\title{
A new class activation functions with application in the theory of impulse techniques
}

\author{
Nikolay Kyurkchiev ${ }^{\mathrm{a}^{*}}$ \\ ${ }^{a}$ Faculty of Mathematics and Informatics, University of Plovdiv Paisii Hilendarski, 24, Tzar Asen Str., 4000 Plovdiv, Bulgaria \\ *Corresponding authorE-mail: nkyurk@math.bas.bg
}

\section{Article Info}

Keywords: "double step" function $\sigma^{*}(t), \sigma^{* *}(t)$-function, emitting chart, Hausdorff distance

2010 AMS: $41 A 46$

Received: 6 May 2018

Accepted: 10 May 2018

Available online: $27 \times x 2018$

\begin{abstract}
In this note we define the new activation functions, based on the well-known hyperbolic tangent and half-hyperbolic tangent activation functions. We consider the Hausdorff distance between the "double step" function $\sigma^{*}(t)$ (resp. function $\sigma^{* *}(t)$ ) and the new classes of activation functions. The results have independent significance in the study of issues related to neural networks and impulse techniques. Numerical examples, illustrating our results are presented using programming environment Mathematica.
\end{abstract}

\section{Introduction}

The typical examples of impulse functions from antenna feeder technique has the following shape (see, Figures 1.1-1.2):

$$
\begin{gathered}
\sigma^{*}(t)=\left\{\begin{array}{l}
1, t \in[1,+\infty) \\
0, t \in[-1,1) \\
-1, t \in(-\infty,-1)
\end{array}\right. \\
\sigma^{* *}(t)=\left\{\begin{array}{l}
1, t \in[-\infty,-1) \cup(1,+\infty) \\
0, t \in[-1,1] .
\end{array}\right.
\end{gathered}
$$

In [3] the following basic problems are considered - approximation of functions and point sets by algebraic and trigonometric polynomials in Hausdorff metric [2] as well as their applications in the field of antenna-feeder technique, analysis and synthesis of antenna patterns and filters, noise minimization by suitable approximation of impulse functions.

The polynomial Hausdorff approximations of the signals of type (1.1)-(1.2) are visualized on Figure 1.3-1.4.

These polynomials play an important role in approximation of antenna factor for scanning of directed chart.

Evidently the task of great difficulty is to determine the coefficients of the polynomials and values of the best Hausdorff approximation. For other results, see [4]-[14], [16].

\section{Main results}

Definition 2.1. [1], [2] The Hausdorff distance (the H-distance) [1] $\rho(f, g)$ between two interval functions $f, g$ on $\Omega \subseteq \mathbb{R}$, is the distance between their completed graphs $F(f)$ and $F(g)$ considered as closed subsets of $\Omega \times \mathbb{R}$. More precisely,

$$
\rho(f, g)=\max \left\{\sup _{A \in F(f)} \inf _{B \in F(g)}\|A-B\|, \sup _{B \in F(g)} \inf _{A \in F(f)}\|A-B\|\right\}
$$




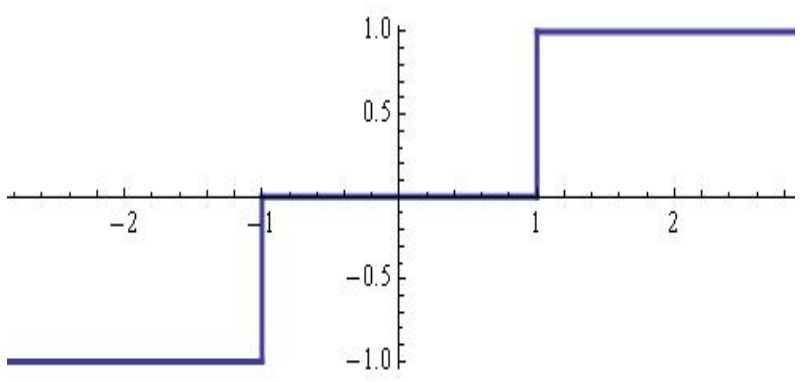

Figure 1.1: The signal of "double step" $\sigma^{*}(t)-$ type.

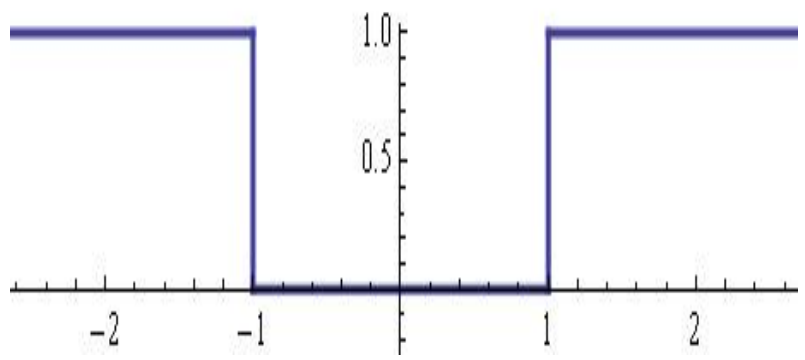

Figure 1.2: The signal of $\sigma^{* *}(t)-$ type.

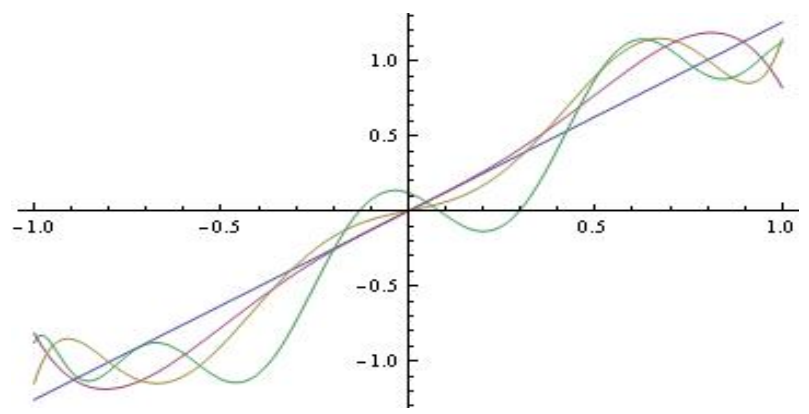

Figure 1.3: The polynomial approximation of the $\sigma^{*}(t)$ - type function.

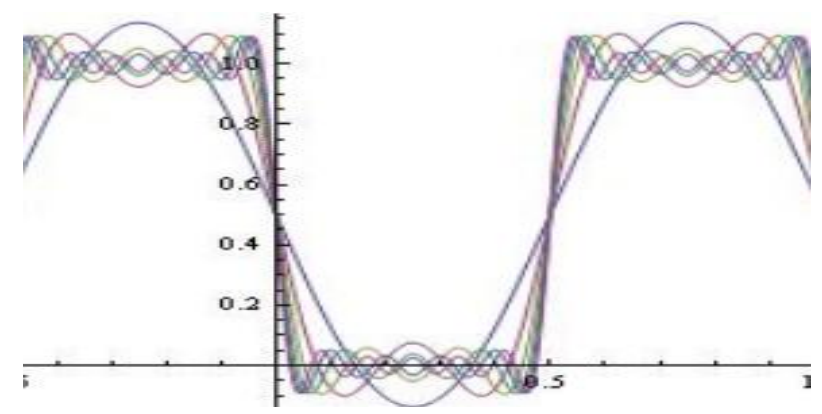

Figure 1.4: The polynomial approximation of the $\sigma^{* *}(t)$ - type function. 


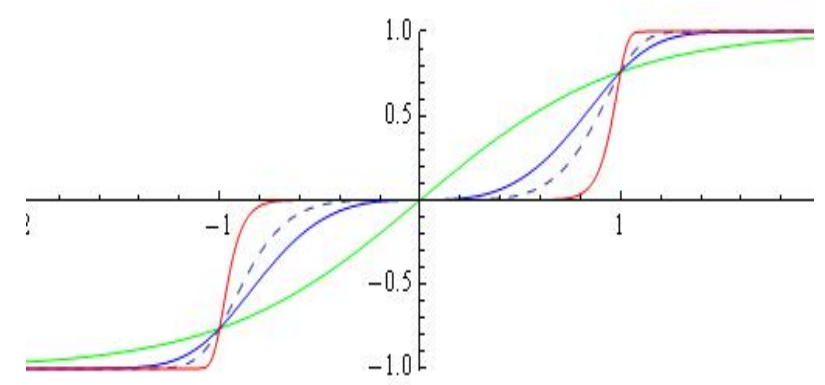

Figure 2.1: The family of activation functions $\varphi^{*}(t)$ for $m=1$ (green); $m=3$ (blue); $m=5$ (dashed); $m=15$ (red); $(\beta=1$ is fixed).

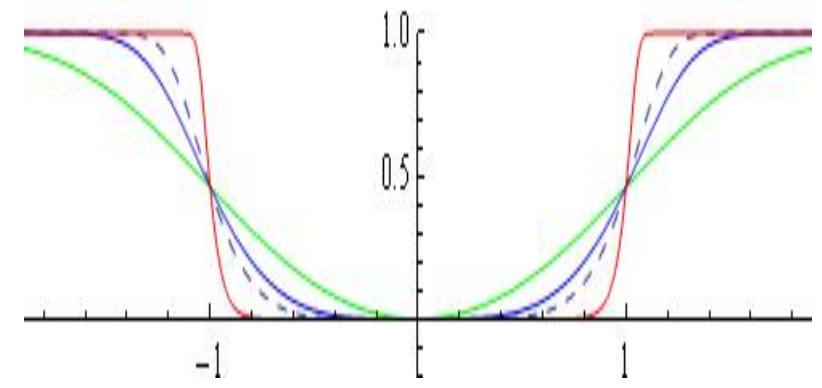

Figure 2.2: The family of activation functions $\varphi^{* *}(t)$ for $m=2$ (green); $m=4$ (blue); $m=6$ (dashed); $m=20$ (red); $(\beta=1$ is fixed).

wherein $\|$.$\| is any norm in \mathbb{R}^{2}$, e. g. the maximum norm $\|(t, x)\|=\max \{|t|,|x|\}$; hence the distance between the points $A=\left(t_{A}, x_{A}\right)$, $B=\left(t_{B}, x_{B}\right)$ in $\mathbb{R}^{2}$ is $\|A-B\|=\max \left(\left|t_{A}-t_{B}\right|,\left|x_{A}-x_{B}\right|\right)$.

Definition 2.2. Define the following "new activation function" as

$$
\varphi^{*}(t)=1-\frac{2 e^{-\beta t^{m}}}{e^{\beta t^{m}}+e^{-\beta t^{m}}}
$$

where $m$ is an odd parameter.

Definition 2.3. Define the following "new half-activation function as:

$$
\varphi^{* *}(t)=\frac{1-e^{-\beta t^{m}}}{1+e^{-\beta t^{m}}}
$$

where $m$ is an even parameter.

The Hausdorff distance $d=d\left(\varphi^{*}(t), \sigma^{*}(t)\right.$ between the activation function $\varphi^{*}(t)$ and the function $\sigma^{*}(t)$ can be calculating by solving nonlinear equation:

$$
\varphi^{*}(1-d)=d
$$

Analogously, for the Hausdorff distance $d=d\left(\varphi^{* *}(t), \sigma^{* *}(t)\right.$ between the activation function $\varphi^{* *}(t)$ and the function $\sigma^{* *}(t)$ we have

$$
\varphi^{* *}(1-d)=d
$$

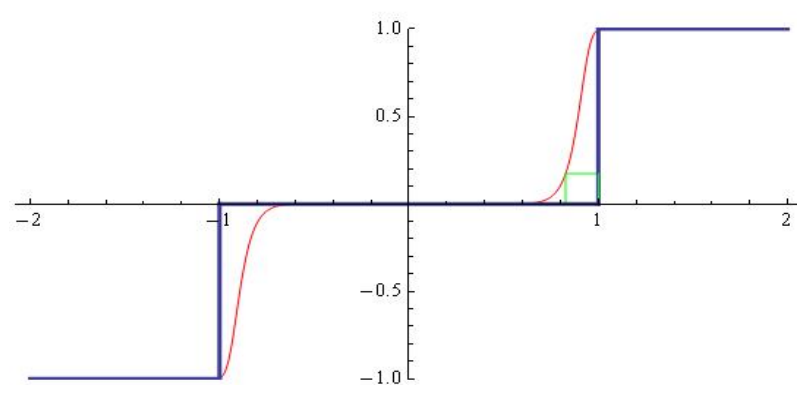

Figure 2.3: The activation functions $\varphi^{*}(t)$ for $\beta=3 ; m=15$; Hausdorff distance $d=0.172738$. 


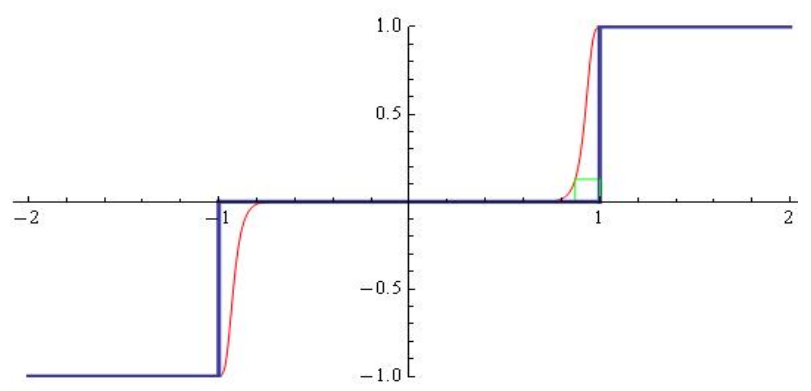

Figure 2.4: The activation functions $\varphi^{*}(t)$ for $\beta=4 ; m=25$; Hausdorff distance $d=0.128338$.

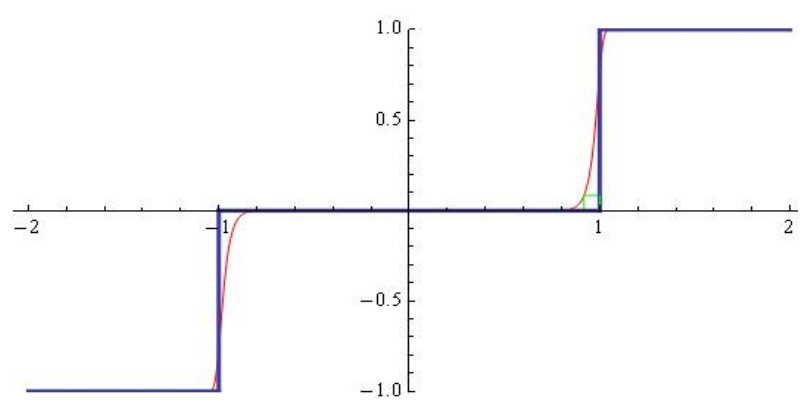

Figure 2.5: The activation functions $\varphi^{*}(t)$ for $\beta=1 ; m=29$; Hausdorff distance $d=0.0824019$

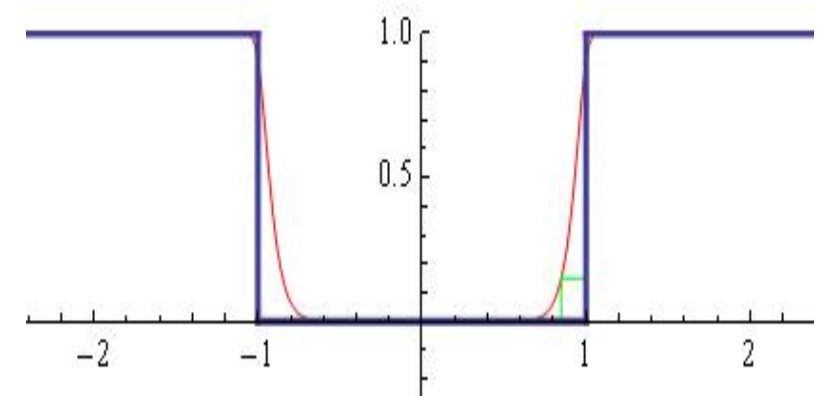

Figure 2.6: The activation functions $\varphi^{* *}(t)$ for $\beta=3 ; m=14$; Hausdorff distance $d=0.15085$.

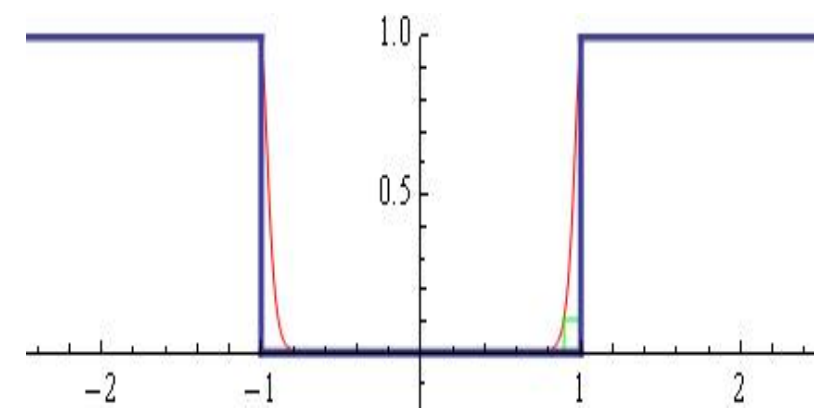

Figure 2.7: The activation functions $\varphi^{* *}(t)$ for $\beta=4 ; m=26$; Hausdorff distance $d=0.106529$. 


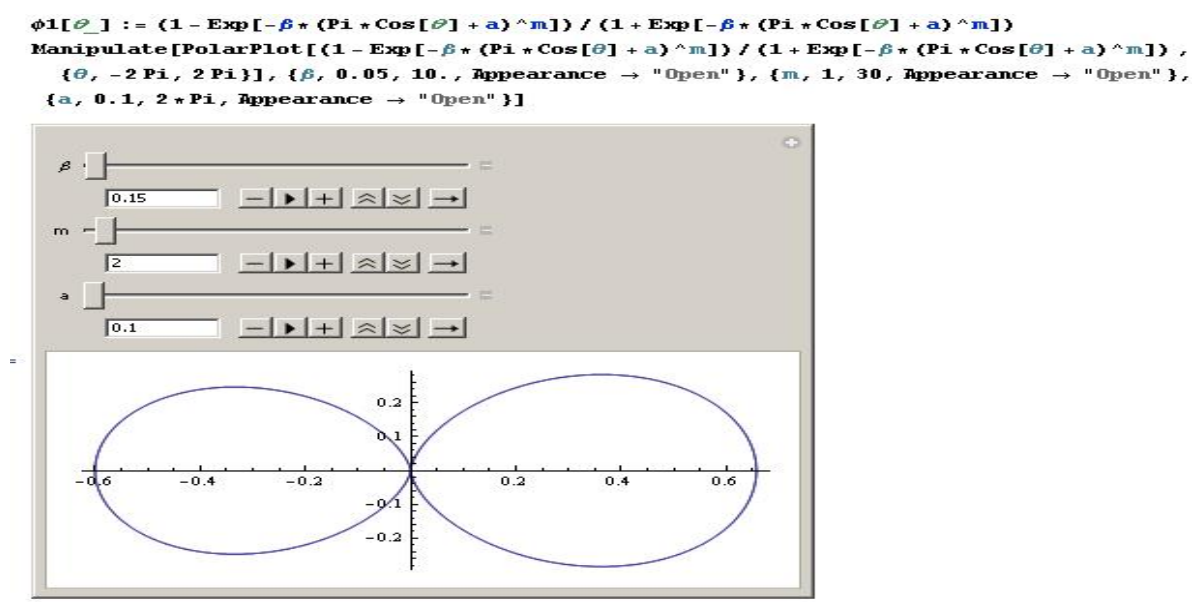

Figure 2.8: Typical emitting chart $\left(\varphi^{* *}(\theta)\right)$ for $\beta=0.15 ; a=0.1 ; m=2$.

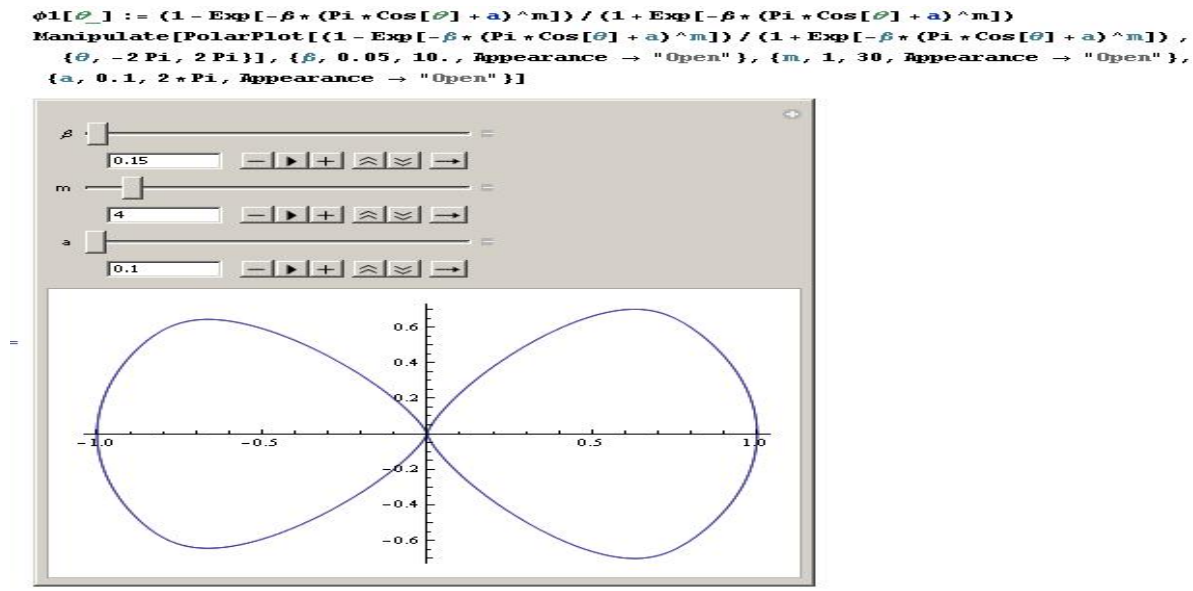

Figure 2.9: Typical emitting chart $\left(\varphi^{* *}(\theta)\right)$ for $\beta=0.15 ; a=0.1 ; m=4$.

Some families of activation functions $\varphi^{*}(t)$ and $\varphi^{* *}(t)$ are visualized on Figures 2.1-2.7.

After the substitution $t=k l \cos \theta+a$, where

$-k=\frac{2 \pi}{\lambda}, \lambda$ is the wave length;

$-a$ is the phase difference;

$-\theta$ is the azimuthal angle;

$-l$ is the distance between the emitters $\left(l=\frac{\lambda}{2}\right.$ is fixed),

the activation function $\varphi^{* *}(t)$ (or emitting chart of antenna factor can be written in the form

$$
\varphi^{* *}(\theta)=\frac{1-e^{-\beta(\pi \cos \theta+a)^{m}}}{1+e^{-\beta(\pi \theta+a)^{m}}}
$$

Typical emitting chart are visualized on Figures 2.8-2.9.

If $l=\lambda$ we have the chart - Figure 2.10.

Of course, the question of the practical realization of the activation functions which are generated as emitting charts remains open.

The mathematical apparatus proposed in the article can be successfully used for imitation and simulation of such charts.

I will explicitly say that the results have independent significance in the study of issues related to impulse technics [3], [15] and neural networks (see, [17]-[21]).

\section{Remarks.}

For the special case $m=1$ we have the following

Theorem [16]. For the Hausdorff distance $d$ between the $s g n$ function and the half-hyperbolic function the following inequalities hold for $\beta \geq 5$ :

$$
d_{l}=\frac{1}{0.5(2+\beta)}<d<\frac{\ln (0.5(2+\beta))}{0.5(2+\beta)}=d_{r}
$$

Following the ideas given in [16], the reader may formulate the corresponding approximation problem for each number $m$. 


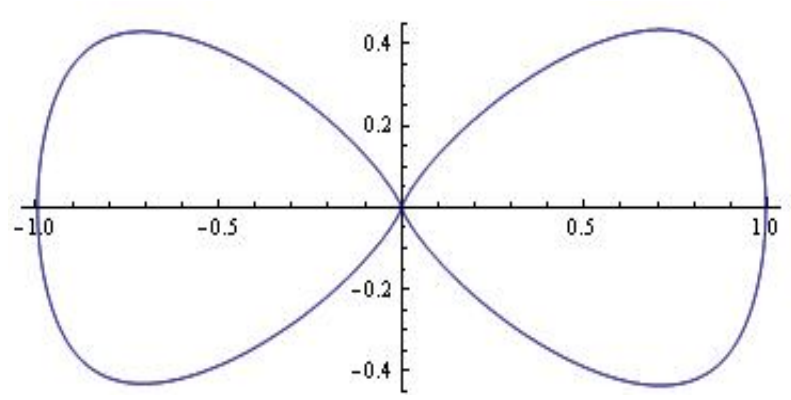

Figure 2.10: The emitting chart for $\beta=0.0001 ; a=0.01 ; m=6 ; l=\lambda$.

\section{Acknowledgements}

This work has been supported by the project FP17-FMI-008 of Department for Scientific Research, Paisii Hilendarski University of Plovdiv.

\section{References}

[1] F. Hausdorff, Set Theory (2 ed.), New York, Chelsea Publ., 1962.

[2] B. SENDOv, Hausdorff Approximations Boston, Kluwer, 1990.

[3] N. KYURKCHIEV AND A. ANDREEV, Approximation and antenna and filter synthesis: Some moduli in programming environment Mathematica, LAP LAMBERT Academic Publishing, Saarbrucken, 2014; ISBN 978-3-659-53322-8.

[4] N. KYURKCHIEV AND BL. SENDOV, Approximation of a class of functions by algebraic polynomials with respect to Hausdorff distance, Ann. Univ. Sofia, Fac. Math., vol. 67, pp 573-579, 1975 (in Bulgarian).

[5] N. KYURKChIEV AND S. MARKOv, On the Hausdorff distance between the Heaviside step function and Verhulst logistic function, J. Math. Chem., vol. 54, no 1, pp 109-119, 2016.

[6] A. ANDREEV AND N. KYURKCHIEv, Approximation of some impulse functions - implementation in programming environment MATHEMATICA, Proceedings of the 43 Spring Conference of the Union of Bulgarian Mathematicians, Borovetz, April 2-6, 2014, pp 111-117.

[7] N. KYURKCHIEV AND S. MARKov, On the numerical approximation of the "cross" set, Ann. Univ. Sofia, Fac. Math., Vol. 66, pp 19-25, 1974 (in Bulgarian).

[8] N. KYURKCHIEV AND A. ANDREEv, Hausdorff approximation of functions different from zero at one point - implementation in programming environment MATHEMATICA, Serdica J. of Computing, vol. 7, no 2, pp 135-142, 2013.

[9] N. KYURKCHIEV AND A. ANDREEV, Synthesis of slot aerial grids with Hausdorff-type directive patterns - implementation in programming environment Mathematica, C.R. Acad. Bulgare Sci., vol. 66, No 11, pp 1521-1528, 2013.

[10] N. KYURKCHIEV, Synthesis of slot aerial grids with Hausdorff type directive patterns, PhD Thesis, Department of Radio-Electronics, VMEI, Sofia, 1979 (in Bulgarian).

[11] BL. SENDOV, H. SCHINEV AND N. KJURKChIEv, Hausdorff-synthesis of aerial grids in scanning the directive diagram, Electropromishlenost $\mathrm{i}$ Priboroostroene, vol. 16, no 5, pp 203-205, 1981 (in Bulgarian).

[12] H. Schinev, N. KJuRKCHIEV AND M. GACHEV, Experimental investigations of slot aerial grids with Hausdorff type directive patterns, Electropromishlenost i Priboroostroene, vol. 14, no 6, pp. 223-224, 1979 (in Bulgarian).

[13] H. Shinev, N. Kyurkchiev, M. Gachev and S. Markov, Application of a class of polynomials of best approximation to linear antenna array synthesis, Izv. VMEI, Sofia, vol. 34, no 1, pp. 1-6, 1975 (in Bulgarian).

[14] A. Golev, T. DJAmiYKov and N. KyURKCHIEV, Sigmoidal functions in antenna-feeder technique, Int. J. of Pure and Appl. Math., vol. 116, no 4, pp 1081-1092, 2017.

[15] N. KyURKChIEv, A. ILIEv AND S. Markov, Some techniques for recurrence generating of activation functions, LAP LAMBERT Academic Publishing, 2017; ISBN 978-3-330-33143-3

[16] V. KYURKCHIEV AND N. KYURKCHIEV, A family of recurrence generated functions based on Half-hyperbolic tangent activation functions, Biomedical Statistics and Informatics, vol. 2, no 3, pp 87-94, 2017.

[17] N. GULIYEV AND V. ISMAILOV, A single hidden layer feedforward network with only one neuron in the hidden layer san approximate any univariate function, Neural Computation, vol. 28, pp 1289--1304, 2016.

[18] D. CostaRELLI AND R. SPIGLER, Approximation results for neural network operators activated by sigmoidal functions, Neural Networks, vol. 44, pp $101-106,2013$.

[19] D. CostaRELLi and G. Vinti, Pointwise and uniform approximation by multivariate neural network operators of the max-product type, Neural Networks, 2016, doi:10.1016/j.neunet.2016.06.002

[20] D. Costarelli AND R. SPIGLER, Solving numerically nonlinear systems of balance laws by multivariate sigmoidal functions approximation, Computational and Applied Mathematics, 2016, doi: 10.1007/s40314-016-0334-8

[21] D. Costarelli AND G. VinTI, Convergence for a family of neural network operators in Orlicz spaces, Mathematische Nachrichten, 2016; doi: 10.1002/mana.20160006 